OPEN ACCESS

Edited by:

Remi Peyronnet

University of Freiburg, Germany

Reviewed by:

Corrado Poggesi,

University of Florence, Italy

Theresia Kraft,

Hannover Medical School, Germany Joachim Meißner,

Hannover Medical School, Germany contributed to the review of TK

*Correspondence:

Tilmann Volk

tilmann.volk@fau.de

Thomas Seidel

thomas.seide/@fau.de

Specialty section: This article was submitted to

Striated Muscle Physiology,

a section of the journal

Frontiers in Physiology

Received: 22 November 2019 Accepted: 17 February 2020

Published: 13 March 2020

Citation:

Abu-Khousa M, Fiegle DJ,

Sommer ST, Minabari G, Milting H, Heim C, Weyand M, Tomasi R, Dendorfer A, Volk $T$ and Seidel $T$ (2020) The Degree of $t$-System Remodeling Predicts Negative Force-Frequency Relationship and Prolonged Relaxation Time in Failing Human Myocardium Front. Physiol. 11:182 doi: 10.3389/fphys.2020.00182

\section{The Degree of t-System Remodeling Predicts Negative Force-Frequency Relationship and Prolonged Relaxation Time in Failing Human Myocardium}

\author{
Maha Abu-Khousa ${ }^{1}$, Dominik J. Fiegle', Sophie T. Sommer', Ghazali Minabari², \\ Hendrik Milting ${ }^{3}$, Christian Heim ${ }^{2}$, Michael Weyand ${ }^{2,4}$, Roland Tomasij,6, \\ Andreas Dendorfer ${ }^{5,7}$, Tilmann Volk ${ }^{1,4 *}$ and Thomas Seidel ${ }^{1,4 *}$
}

1 Institute of Cellular and Molecular Physiology, Friedrich-Alexander-Universität Erlangen-Nürnberg, Erlangen, Germany, ${ }^{2}$ Department of Cardiac Surgery, Friedrich-Alexander-Universität Erlangen-Nürnberg, Erlangen, Germany, ${ }^{3}$ Erich and Hanna Klessmann Institute, Clinic for Thoracic and Cardiovascular Surgery, Heart and Diabetes Center NRW, Ruhr University Bochum, Bad Oeynhausen, Germany, ${ }^{4}$ Muscle Research Center Erlangen (MURCE), Friedrich-Alexander-Universität Erlangen-Nürnberg, Erlangen, Germany, ${ }^{5}$ Walter Brendel Centre of Experimental Medicine, University Hospital, LMU Munich, Munich, Germany, ${ }^{6}$ Department of Anaesthesiology, University Hospital, LMU Munich, Munich, Germany, ${ }^{7}$ German Center for Cardiovascular Research (DZHK), Partner Site Munich Heart Alliance, Munich, Germany

The normally positive cardiac force-frequency relationship (FFR) becomes flat or negative in chronic heart failure (HF). Here we explored if remodeling of the cardiomyocyte transverse tubular system (t-system) is associated with alterations in FFR and contractile kinetics in failing human myocardium. Left-ventricular myocardial slices from 13 failing human hearts were mounted into a biomimetic culture setup. Maximum twitch force (F), 90\% contraction duration (CD90), time to peak force (TTP) and time to relaxation (TTR) were determined at $37^{\circ} \mathrm{C}$ and $0.2-2 \mathrm{~Hz}$ pacing frequency. $\mathrm{F}_{1 \mathrm{~Hz}} / \mathrm{F}_{0.5 \mathrm{~Hz}}$ and $\mathrm{F}_{2 \mathrm{~Hz}} / \mathrm{F}_{0.5 \mathrm{~Hz}}$ served as measures of $\mathrm{FFR}$, intracellular cardiomyocyte t-tubule distance $(\Delta T T)$ as measure of t-system remodeling. Protein levels of SERCA2, NCX1, and PLB were quantified by immunoblotting. $\mathrm{F}_{1 \mathrm{~Hz}} / \mathrm{F}_{0.5 \mathrm{~Hz}}\left(R^{2}=0.82\right)$ and $\mathrm{F}_{2 \mathrm{~Hz}} / \mathrm{F}_{0.5 \mathrm{~Hz}}\left(R^{2}=0.5\right)$ correlated negatively with $\Delta T$, i.e., samples with severe t-system loss exhibited a negative FFR and reduced myocardial wall tension at high pacing rates. PLB levels also predicted $\mathrm{F}_{1 \mathrm{~Hz}} / \mathrm{F}_{0.5 \mathrm{~Hz}}$, but to a lesser degree $\left(R^{2}=0.49\right)$, whereas $\mathrm{NCX} 1$ was not correlated $\left(R^{2}=0.02\right)$. CDgo correlated positively with $\Delta T\left(R^{2}=0.39\right)$ and negatively with SERCA2/PLB $\left(R^{2}=0.42\right)$, indicating that both the t-system and SERCA activity are important for contraction kinetics. Surprisingly, $\Delta T$ was not associated with TTP $\left(R^{2}=0\right)$ but rather with TTR $\left(R^{2}=0.5\right)$. This became even more pronounced when interaction with NCX1 expression was added to the model $\left(R^{2}=0.79\right)$, suggesting that t-system loss impairs myocardial relaxation especially when NCX1 expression is low. The degree of t-system remodeling predicts FFR inversion and contraction slowing in failing human myocardium. Moreover, together with NCX, the t-system may be important for myocardial relaxation.

Keywords: heart failure, cardiac excitation-contraction coupling, cardiac remodeling, human heart, force frequency relationship, transverse tubular system 


\section{INTRODUCTION}

The myocardial force-frequency relationship (FFR), also referred to as staircase phenomenon, describes the frequency-dependent change of cardiac contractile force in the absence of other external stimuli and is therefore considered as an intrinsic regulatory mechanism of the heart. Most mammals, including humans, exhibit a positive FFR in the physiological range of heart rates (Janssen and Periasamy, 2007). Because heart rate is increased predominantly at the expense of ventricular filling time, enddiastolic volume decreases at high heart rates. Therefore, systolic force and contraction velocity must increase to maintain a high stroke volume during physical activity (Higginbotham et al., 1986), which, in addition to sympathetic activation, may be facilitated by a positive FFR.

It is widely accepted that dynamic changes in intracellular $\mathrm{Ca}^{2+}$ underlie the FFR. At high heart rates, increased influxes of $\mathrm{Ca}^{2+}$ and $\mathrm{Na}^{+}$due to more frequent action potentials cause respective rises in intracellular concentrations. This leads to increased $\mathrm{Ca}^{2+}$ load of the sarcoplasmic reticulum (SR), because more cytosolic $\mathrm{Ca}^{2+}$ is available for the SR $\mathrm{Ca}^{2+}$ ATPase (SERCA). Additionally, the increased amount of intracellular $\mathrm{Na}^{+}$reduces the driving force for the sodiumcalcium exchanger (NCX). As a consequence, the relative contribution of SERCA to cytosolic $\mathrm{Ca}^{2+}$ removal increases. The overall effect is an increase in contractile force. However, while this is one common explanation for the positive FFR in healthy myocardium, additional mechanisms have been suggested (Endoh, 2004; Janssen and Periasamy, 2007), and it remains not completely understood how these mechanisms are altered in diseased myocardium.

The normally positive FFR becomes flat or negative in failing human myocardium (Mulieri et al., 1992). This is thought to contribute to poor cardiac function in heart failure patients, especially under conditions of cardiac stress (Hasenfuss et al., 1994a). It has been shown that altered expression levels and activities of $\mathrm{Ca}^{2+}$ cycling proteins, such as SERCA (Hasenfuss et al., 1994b; Munch et al., 2000), PLB (Hasenfuss et al., 1996; Brixius et al., 2003) and NCX (Hasenfuss et al., 1999) are associated with the negative FFR in human failing myocardium.

However, another important requirement for efficient $\mathrm{Ca}^{2+}$ cycling and excitation-contraction coupling in ventricular cardiomyocytes is the transverse tubular system (t-system). The $\mathrm{t}$-system is formed by regularly arranged membrane tubules running from the surface into the cytosol, where they form close junctions with the SR. By bringing L-type $\mathrm{Ca}^{2+}$ channels close to ryanodine receptors in the $\mathrm{SR}, \mathrm{Ca}^{2+}$-induced $\mathrm{Ca}^{2+}$ release is largely facilitated. Additionally, t-tubules contain a high density of NCX, which may facilitate $\mathrm{Ca}^{2+}$ extrusion across the cell membrane (Thomas et al., 2003). A common feature of failing myocardium is remodeling and loss of the t-system (Kostin et al., 1998). The remodeling includes changes in t-tubule structure and reduced density of the t-system and has been associated with poor cardiac function and impaired recovery (Seidel et al., 2017a). Because t-system remodeling impairs intracellular $\mathrm{Ca}^{2+}$ cycling and causes slowed as well as reduced $\mathrm{Ca}^{2+}$ release (Louch et al., 2004; Seidel et al., 2019), we hypothesized that t-system remodeling may be associated with FFR inversion and altered contractile kinetics.

In this report we explored the correlation of t-system remodeling with the FFR and contraction kinetics in myocardial tissue slices from failing human hearts. We show that high degrees of t-system remodeling are associated with negative FFR and slowed contraction. Furthermore, we show that an interaction between NCX expression and t-system density may predict myocardial relaxation time.

\section{METHOD}

Methods are described in detail in the Supplementary Material.

\section{Human Cardiac Samples}

Transmural myocardial samples were collected from end-stage failing hearts with reduced ejection fraction from the leftventricular (LV) apical core during implantation of mechanical assist devices or from the free LV wall of explanted hearts. An overview of the patients is displayed in Supplementary Table S1. Collection and use of human cardiac tissue samples was approved by the Institutional Review Boards of the University of Erlangen-Nürnberg, the Ruhr-University Bochum and the Ludwig-Maximilian University Munich. Studies were conducted according to Declaration of Helsinki principles. Patients gave their written informed consent prior to tissue collection.

\section{Sample Preparation and Analysis of Contractile Parameters}

Following a published method for stable cultivation of human myocardium (Fischer et al., 2019), myocardial slices of $300 \mu \mathrm{m}$ thickness and approximately $5 \mathrm{~mm} \times 5 \mathrm{~mm}$ length and width were cut with a high-precision vibratome (Leica VT1200S) and installed into biomimetic cultivation chambers at $37^{\circ} \mathrm{C}$. Electrodes and a force-transducing wire included in the chambers allowed for continuous electrical stimulation and monitoring of contractile force during auxotonic (elastic) contraction (see Supplementary Figure S1). The sarcomere length at different degrees of preload was assessed in initial experiments by $\alpha$-actinin staining. Preload was then set to $1.5 \mathrm{mN} / \mathrm{mm}^{2}$ in all slices to obtain a resting sarcomere length between 2.0 and $2.1 \mu \mathrm{m}$ (see Supplementary Figure S2), which is close to the optimum suggested for stable cultivation of myocardial tissue (Watson et al., 2019).

For all cultivated myocardial slices, the baseline stimulation rate was $0.5 \mathrm{~Hz}$. The FFR and single-contraction parameters were assessed within $24 \mathrm{~h}$ after installation, using a protocol with intervals of $120 \mathrm{~s}$ duration and increasing stimulation frequencies of $0.2,0.5,1$, and $2 \mathrm{~Hz}$. Contractions during the last $30 \mathrm{~s}$ of each interval were analyzed with custom-written software scripts (Matlab 2019a). For each contraction, maximal force $\left(F_{\max }\right)$, time to peak (TTP), time to relaxation (TTR) and $90 \%$ contraction duration $\left(\mathrm{CD}_{90}\right)$ were analyzed and then averaged over the $30 \mathrm{~s}$ interval. Actively developed wall tension (T) was calculated by dividing the developed force (F) by the cross-sectional area (A): $T=\mathrm{F} / \mathrm{A}$. To estimate $\mathrm{A}$, the width of the slices $(5 \mathrm{~mm})$ was 
multiplied with the nominal thickness $(300 \mu \mathrm{m})$ minus $50 \mu \mathrm{m}$, assuming that the very top and bottom myocyte layers were damaged during the slicing: $A=5 \mathrm{~mm} \times 0.25 \mathrm{~mm}=1.25 \mathrm{~mm}^{2}$.

\section{Immunostaining and Confocal Imaging}

Neighboring tissue slices of the functionally assessed slices were fixed with $2 \%$ PFA immediately after slicing and stained with AF647-conjugated wheat germ agglutinin (WGA, Thermo Fisher, W32466) to visualize the extracellular matrix, surface sarcolemma and t-tubules. RyR2 (Thermo Fisher, MA3-916) or $\alpha$-actinin (A7811, Sigma) were co-stained with AF488 (A21121, Thermo Fisher). For the validation of the WGA staining, human tissue was co-stained with a mouse monoclonal anti-caveolin-3 antibody (sc-5310, Santa Cruz, CA, United States) and WGA (see Supplementary Figure S3).

The samples were mounted in Fluoromount G (Sigma, F4680) and $3 \mathrm{D}$ confocal image stacks $(1280 \times 1280 \times 300$ voxels, voxel size $0.1 \times 0.1 \times 0.1 \mu^{3}$ ) were acquired with a Zeiss LSM780 confocal microscope with a $63 \times$ oil immersion lens. At least 3 image stacks from each sample were recorded from randomly chosen regions. Additionally, two-dimensional confocal tile scans $\left(1-5 \mathrm{~mm}^{2}\right)$ were acquired with a pixel size of $0.15 \times 0.15 \mathrm{\mu m}^{2}$. The researchers were blinded against functional parameters.

\section{Image Analysis}

Applying published methods (Seidel et al., 2013, 2016, 2017a,b), confocal image stacks were corrected for depth-dependent attenuation and then filtered, deconvolved and segmented, using histogram-based thresholds. By applying automated analysis scripts, the cardiomyocyte t-system could be distinguished from the outer sarcolemma and interstitial space, using either RyR or $\alpha$-actinin as cardiomyocyte marker (Seidel et al., 2017a,b). Sarcomere length was determined by 2D Fourier transformation of $\alpha$-actinin images and identification of the maximum in the power spectrum within a spatial frequency of $1 / 2.5$ to $1 / 1.5 \mathrm{\mu m}^{-1}$ (Seidel et al., 2017b). As a measure of t-system remodeling, we calculated the mean intracellular distance to the closest t-tubule $(\Delta \mathrm{TT})$, using the threedimensional Euclidean distance transform of the extracted t-tubule signal and the cardiomyocyte mask (mean $\Delta \mathrm{TT}$ from at least 3 image stacks per myocardial sample). To assess cardiomyocyte disarray, large area confocal tile scans (1$5 \mathrm{~mm}^{2}$ ) were noise-filtered, deconvolved and then subjected to a watershed-based cardiomyocyte segmentation method published previously (Seidel et al., 2013). In brief, the watershed seeds were generated from the distance transform of the WGA image, followed by a morphological watershed segmentation with the distance transform as gradient image. Subsequently, segments with low contact area to the WGA signal were removed and the watershed run again. This process was iterated until most myocytes contained only one segment. The main axis orientation of myocytes was then determined by calculating the eigenvectors of the covariance matrix (2nd order central image moments) of each segment as described (Lackey et al., 2011).

\section{Western Blotting}

Small transmural tissue blocks from each sample were frozen at $-80^{\circ} \mathrm{C}$ and used later for Western blot analysis. Primary antibodies against SERCA2, NCX1, PLB and phosphorylated PLB (pS16 and pT17) were used. Densitometric measurements were performed with ImageJ/Fiji software and normalized to the geometric means of Ponceau staining and GAPDH. To make samples from different blots comparable, we additionally normalized to a reference, which was added to each gel. A more detailed description of Western blotting, including antibody specifications, is available in the Supplementary Material.

\section{Statistics}

For each myocardial sample, contractile parameters (force, TTR, TTP, CD90, FFR) were assessed simultaneously in 2-8 neighboring tissue slices and the medians used as representative measures for subsequent statistical analyses. Correlation between parameters was tested by linear regression (fitlm function, Matlab version R2019a) with least squares fitting. $P$-values reported for linear models were obtained from an $F$-test against the corresponding constant model (intercept only, i.e., no effect of the predicting variable). $P$-values below 0.05 (type I error) were considered significant. The unpaired two-tailed student's $t$-test was used to compare means between different groups. The HolmBonferroni method was used to correct $P$-values for multiple comparisons. If not indicated otherwise, data are reported as mean \pm standard error (SEM).

\section{RESULTS}

\section{Variable t-System Density and Frequency Response Amongst Myocardial Samples}

We obtained LV myocardial samples from 13 patients suffering from end-stage heart failure (LV ejection fraction $20.6 \pm 7 \%$, Supplementary Table S1) undergoing either LVAD implantation $(n=5)$ or heart transplantation $(n=8)$. In vibratome-cut myocardial slices of $300 \mu \mathrm{m}$ thickness we assessed the FFR over a frequency range from 0.2 to $2 \mathrm{~Hz}$ and stained the extracellular matrix and cell membranes including the t-system with WGA. Reliable staining of t-tubules with WGA was verified by co-staining of caveolin-3, a commonly used membrane marker of cardiomyocytes (Supplementary Figure S3). Threedimensional confocal microscopic images showed a substantial variability in the degree of t-tubular remodeling between samples from different patients (Figures 1A-D). While some samples presented with a dense and well-preserved t-system and, accordingly, low intracellular t-tubule distances $(\Delta \mathrm{TT})$ (Figures 1A,B), others displayed severe t-system loss, resulting in high $\triangle \mathrm{TT}$ (Figures 1C,D). Inspection of the corresponding FFRs revealed positive relationships in samples with low $\Delta \mathrm{TT}$, i.e., preserved t-system (Figure 1E). In these samples the maximum twitch force increased with increasing stimulation frequency. In contrast, samples with high $\Delta \mathrm{TT}$, i.e., t-system loss, exhibited a flat response at lower frequencies and a clearly negative 

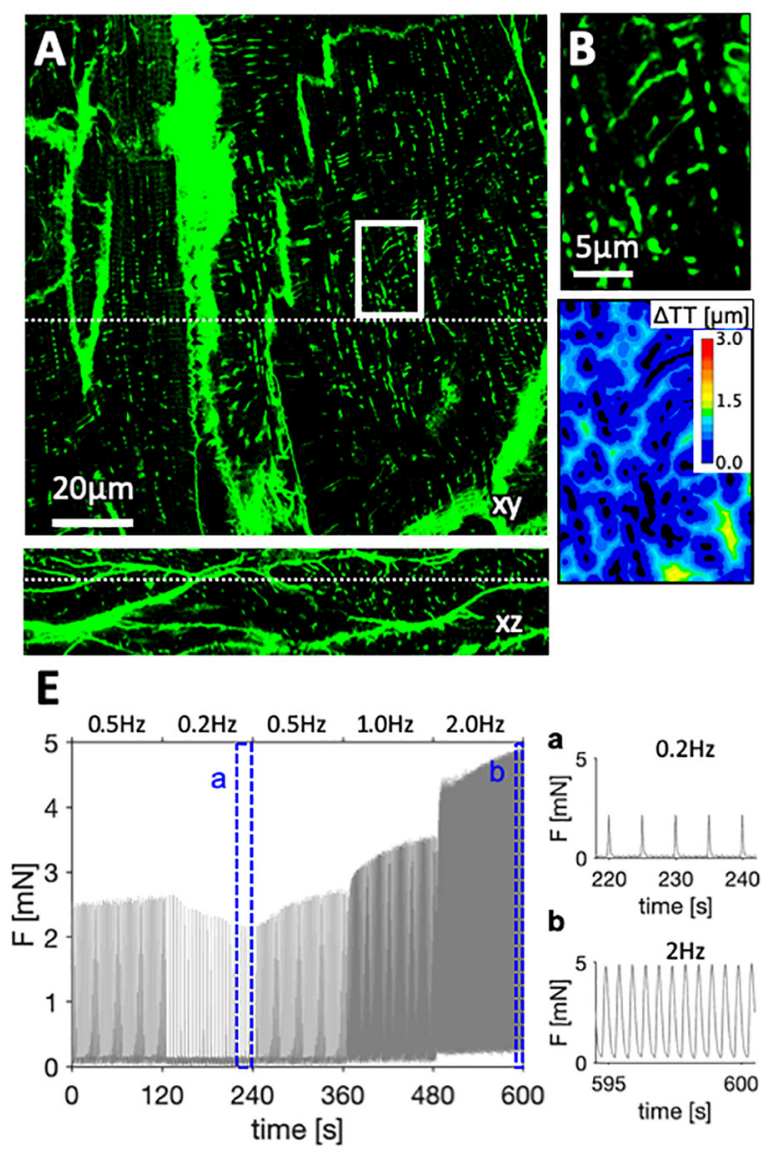

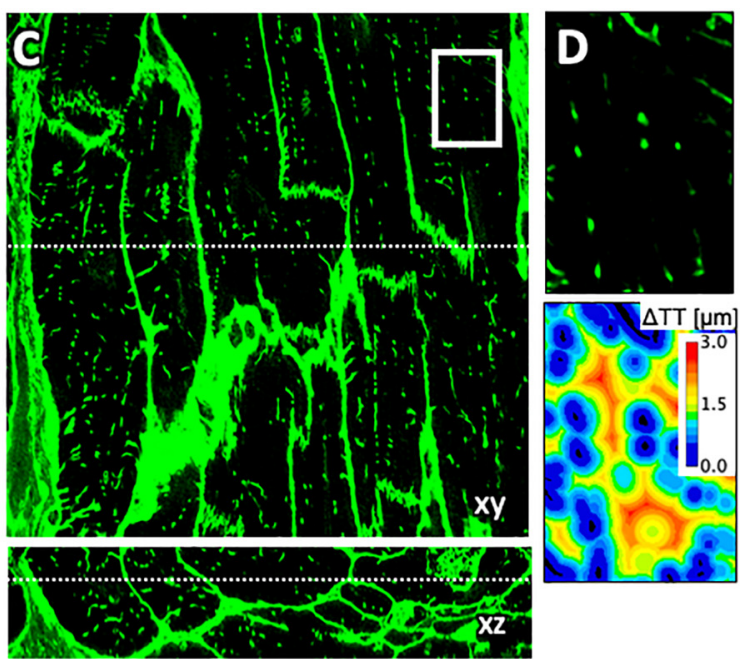

$\mathbf{F}$

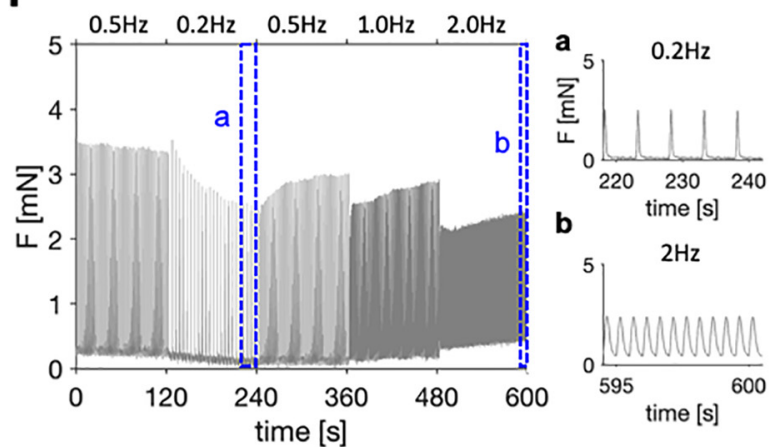

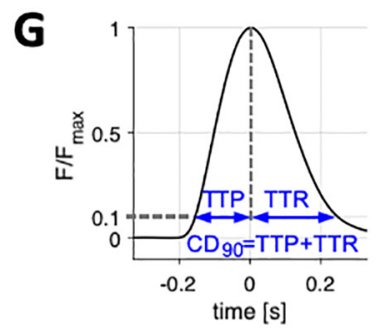

H $\begin{aligned} & \text { dense t-system (mean } \Delta \mathrm{TT}=0.83 \mu \mathrm{m} \text {, shown in } \mathrm{A}, \mathrm{B}, \mathrm{E}) \\ & \text { sparse t-system (mean } \Delta \mathrm{TT}=1.06 \mu \mathrm{m} \text {, shown in } \mathrm{C}, \mathrm{D}, \mathrm{F} \text { ) }\end{aligned}$
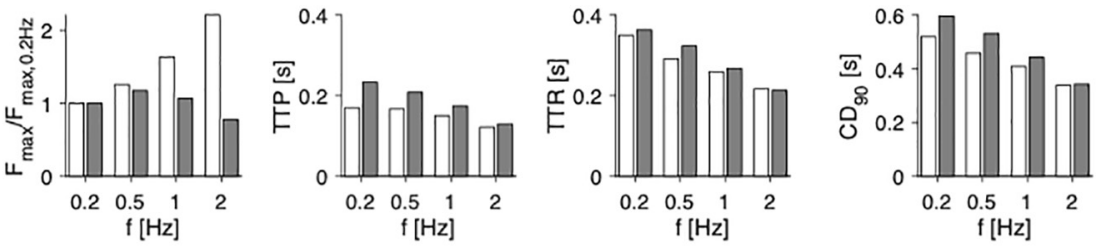

FIGURE 1 | Examples of tissue slices from myocardial specimens with high and low degree of t-system remodeling and corresponding force-frequency relationship. Three-dimensional confocal images in xy and xz views of left-ventricular myocardial tissue slices stained with wheat germ agglutinin from (A,B) specimen with dense t-system, and (C,D) specimen with sparse t-system. B and D show the respective magnifications of the boxed regions in $A$ and $C$, and the corresponding $3 D$ distance maps (color-coded distance to closest t-tubule, $\Delta T$ ). Dotted lines in A and $\mathrm{C}$ indicate the optical sections of the xy and $\mathrm{xz}$ views. (E,F) Twitch forces of myocardial slices from the specimens shown in A and C, respectively, stimulated with increasing pacing frequency. The highlighted intervals (a, b) are magnified. (G) Exemplary trace of one twitch, normalized to its maximum force $\left(F_{\max }\right)$ with schematic of assessed contraction parameters: time to peak (TTP), time to relaxation (TTR) and 90\% contraction duration (CD90). (H) Normalized $F_{\max }$, TTP, TTR and CD9o at the assessed frequencies in the two examples shown in A (example with low $\Delta T$, i.e., dense t-system) and in $\mathrm{C}$ (example with high $\Delta T$, i.e., sparse t-system). Scale bar in $\mathrm{A}$ also applies to $\mathrm{C}$, scale bar in $\mathrm{B}$ also applies to $\mathrm{D}$.

response at higher frequencies (Figure 1F). We compared selfnormalized forces and the following parameters of contraction kinetics: time to peak force (TTP), time to relaxation (TTR) and $90 \%$ contraction duration $\left(\mathrm{CD}_{90}\right.$, Figure 1G). Figure $\mathbf{1 H}$ shows that the maximum force in the sample with preserved t-system (low $\Delta \mathrm{TT}$ ) increased more than twofold from 0.2 to $2 \mathrm{~Hz}$, but decreased by approximately $25 \%$ in the sample with reduced t-system density (high $\Delta$ TT). TTP, TTR, and $\mathrm{CD}_{90}$ fitted well to values reported in other studies (Mulieri et al., 1992; Rossman et al., 2004) and decreased consistently in both samples with increasing pacing frequency, but higher values of TTP, TTR, and $\mathrm{CD}_{90}$ indicated slowed contraction in the example with $\mathrm{t}$-system loss, especially at low pacing frequencies. In conclusion, the results shown in Figure $\mathbf{1}$ illustrate that the degree of t-system loss might be related to the FFR and contraction kinetics. 


\section{The Degree of $t$-System Remodeling Predicts the Degree of FFR Inversion}

In order to evaluate if $\Delta \mathrm{TT}$ may generally predict the FFR in failing human myocardium, we performed linear regression including the functional and histological data from all available samples. Mean $\Delta \mathrm{TT}$ values, acquired from at least 3 confocal image stacks at randomly chosen regions of the tissue slices, ranged from approximately 0.8 to $1.5 \mu \mathrm{m}$. This most likely reflects the high regional heterogeneity of t-system loss across the left ventricle in failing hearts, as previously described (Crossman et al., 2015), see discussion. As measures of the FFR, we used the ratio between the maximum force amplitudes at 1 and $0.5 \mathrm{~Hz}$ $\left(\mathrm{F}_{1 \mathrm{~Hz}} / \mathrm{F}_{0} \cdot 5 \mathrm{~Hz}\right)$ as well as 2 and $0.5 \mathrm{~Hz}\left(\mathrm{~F}_{2 \mathrm{~Hz}} / \mathrm{F}_{0.5 \mathrm{~Hz}}\right)$. Values above or below 1 thus indicate a positive or negative FFR, respectively. Figure $2 \mathrm{~A}$ shows the linear regression of $\mathrm{F}_{1 \mathrm{~Hz}} / \mathrm{F}_{0} \cdot 5 \mathrm{~Hz}$ over $\Delta \mathrm{TT}$, which yielded a negative and highly significant relationship between the both parameters. This means that with increasing $\Delta$ TT the FFR became more negative $\left(R^{2}=0.82, p<0.0001\right)$. The model predicted inversion of the $\mathrm{FFR}\left(\mathrm{F}_{1 \mathrm{~Hz}} / \mathrm{F}_{0} \cdot 5 \mathrm{~Hz} \leq 1\right)$ at $\Delta \mathrm{TT} \geq 1.05 \mu \mathrm{m}$. Thus, we divided the samples into two groups of similar size: samples with low to moderate t-system loss $(\Delta \mathrm{TT}<1.05 \mu \mathrm{m}, n=6)$, and samples with severe t-system loss $(\Delta \mathrm{TT} \geq 1.05 \mu \mathrm{m}, n=7)$. We performed a $t$-test to compare the corresponding means of $\mathrm{F}_{1 \mathrm{~Hz}} / \mathrm{F}_{0} \cdot 5 \mathrm{~Hz}$ (Figure 2B). Mean $\mathrm{F}_{1 \mathrm{~Hz}} / \mathrm{F}_{0} \cdot 5 \mathrm{~Hz}$ in the group with $\Delta \mathrm{TT}<1.05 \mu \mathrm{m}$ was $1.08 \pm 0.05$, indicating a slightly positive FFR, whereas the group with $\Delta \mathrm{TT} \geq 1.05 \mu \mathrm{m}$ exhibited a markedly negative FFR $(0.80 \pm 0.05, p<0.01)$. When analyzing the relationship between $\Delta \mathrm{TT}$ and $\mathrm{F}_{2 \mathrm{~Hz}} / \mathrm{F}_{0} \cdot 5 \mathrm{~Hz}$ (Figures 2C,D), we found very similar results, although the coefficient of determination was not as high $\left(R^{2}=0.5, p<0.01\right)$. In accordance with these findings, mean maximum wall tension did not differ between the two groups at 0.2 and $0.5 \mathrm{~Hz}$ pacing frequency, but was distinctively higher at 1 and $2 \mathrm{~Hz}$ in the group with $\Delta \mathrm{TT}<1.05 \mu \mathrm{m}$ than with $\Delta \mathrm{TT} \geq 1.05 \mu \mathrm{m}(2.01 \pm 0.46$ vs. $0.72 \pm 0.16$ and $1.93 \pm 0.44$ vs. $0.71 \pm 0.2$, respectively, in $\mathrm{mN} / \mathrm{mm}^{2}, p<0.05$ ) (Figure $2 \mathrm{E}$ ). In summary, these data indicate that in human failing myocardium a frequency-related increase in contractile force may require a dense $\mathrm{t}$-system and that the degree of $\mathrm{t}$-system remodeling predicts to a substantial extent a functional decline in FFR.

\section{Association of $\mathrm{Ca}^{2+}$ Cycling Proteins With FFR}

Next, to investigate if $\mathrm{Ca}^{2+}$ cycling proteins reported to correlate with the FFR of human failing myocardium predict the FFR to a similar extent as the degree of t-system remodeling, we used Western blotting to quantify protein expression levels of NCX1, SERCA2, and PLB, including its phosphorylated forms pS16 and pT17 (Figure 3A). In 4 out of the 13 samples, the amount of available tissue was not sufficient for protein analysis. Probing for NCX1 yielded clear bands at $120 \mathrm{kDa}$ and $70 \mathrm{kDa}$, as described in other studies using the same primary antibody. Because both bands have been shown to represent functional forms of NCX1, we included both in our analyses (McDonald et al., 2000). We found that the expression of $\mathrm{Ca}^{2+}$ cycling proteins varied considerably and that the FFR did not correlate
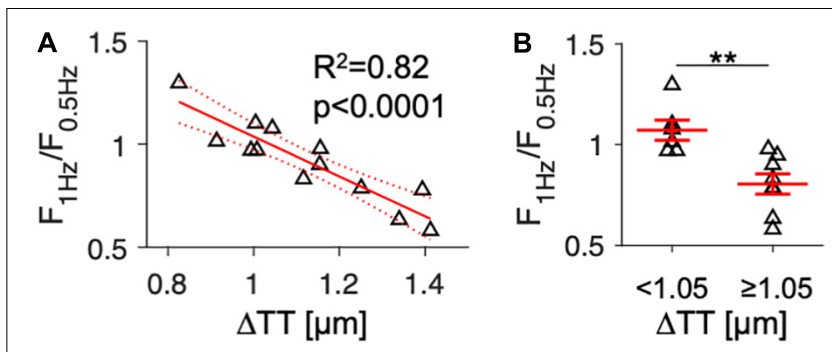

C

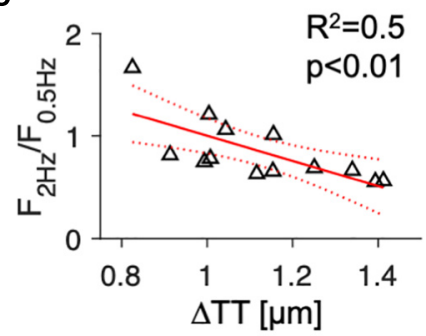

D

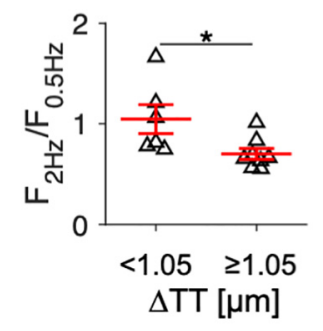

E

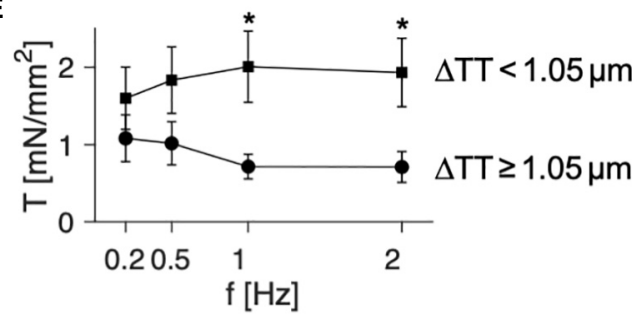

FIGURE 2 | Association of the force-frequency relationship and wall tension with cardiomyocyte t-tubule distance in tissue slices from human failing hearts ( $n=13$ ). (A) Linear model showing the correlation of force at $1 \mathrm{~Hz}$ relative to force at $0.5 \mathrm{~Hz}$ pacing rate $\left(\mathrm{F}_{1 \mathrm{~Hz}} / \mathrm{F}_{0.5 \mathrm{~Hz}}\right)$ with t-tubule distance $(\Delta T \mathrm{~T}) . \mathrm{R}^{2}$ is the coefficient of determination, $\mathrm{p}$ indicates the probability of type I error of linear regression. Dotted lines indicate 95\% confidence intervals. (B) $\mathrm{F}_{1 \mathrm{~Hz}} / \mathrm{F}_{0.5 \mathrm{~Hz}}$ in specimens with low $(<1.05 \mu \mathrm{m}, n=7)$ and high $\Delta T \mathrm{~T}(\geq 1.05 \mu \mathrm{m}, n=7)$. Note that low $\Delta T$ indicates high t-tubule density. (C) Correlation of force at $2 \mathrm{~Hz}$ relative to force at $0.5 \mathrm{~Hz}$ pacing rate $\left(\mathrm{F}_{2 \mathrm{~Hz}} / \mathrm{F}_{0.5 \mathrm{~Hz}}\right)$ with $\Delta T$. (D) $\mathrm{F}_{2 \mathrm{~Hz}} / \mathrm{F}_{0.5 \mathrm{~Hz}}$ in specimens with low and high $\Delta T$. (E) Mean wall tension (T) developed actively during contraction in specimens with low (squares) and high $\Delta T$ (circles) at $0.2-2 \mathrm{~Hz}$ pacing frequency (f). ${ }^{*} p<0.05$, ${ }^{* *} p<0.01$ (unpaired, two-tailed $t$-test, multiple comparison adjustment in $\mathrm{E}$ ), error bars indicate SEM.

with NCX1 or SERCA2 (Figures 3B,C). However, $\mathrm{F}_{1 \mathrm{~Hz}} / \mathrm{F}_{0} \cdot 5 \mathrm{~Hz}$ showed a significant negative correlation with total PLB. Samples with negative FFR displayed higher levels of PLB than samples with positive FFR $\left(R^{2}=0.49, p<0.05\right.$, Figure 3D). Using the SERCA2/PLB ratio improved the prediction only marginally $\left(R^{2}=0.52, p<0.05\right.$, Figure $\left.3 \mathrm{E}\right)$. We also explored whether the extent of PLB inhibition by phosphorylation through PKA (PLBpS16) or CAMKII (PLBpT17) was associated with the FFR. While PLBpS16 and the PLBpS16/PLB ratio did not predict the FFR, the PLBpT17/PLB ratio showed a moderate positive association $\left(R^{2}=0.44, p<0.05\right.$, Supplementary Table S2). These results indicate that SERCA2 or NCX1 expression alone were poor predictors of the FFR and that proteins and measures reflecting SERCA2 activity (PLB, SERCA2/PLB ratio, 
A

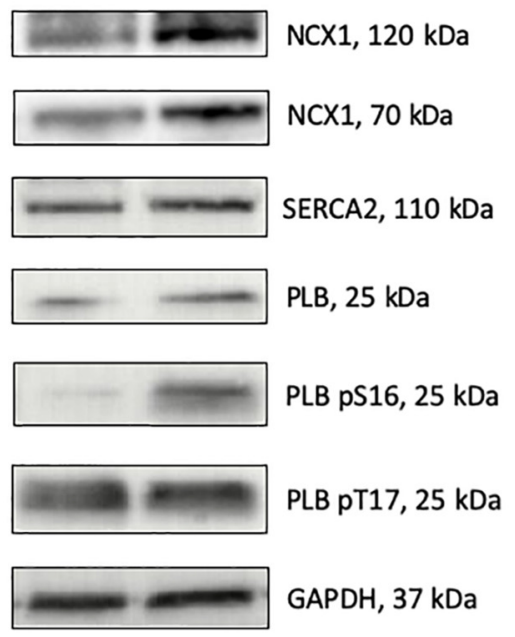

B

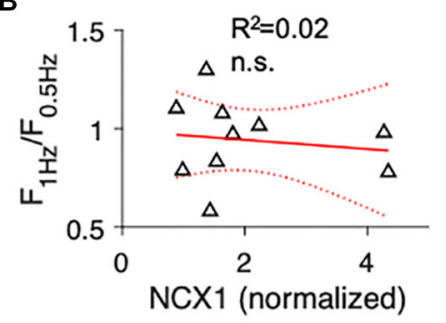

D

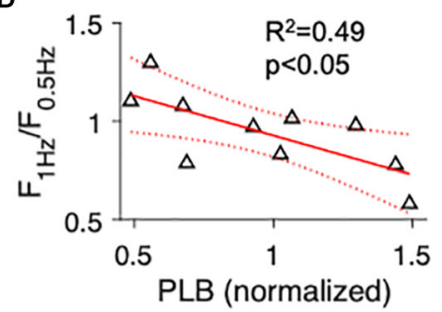

C

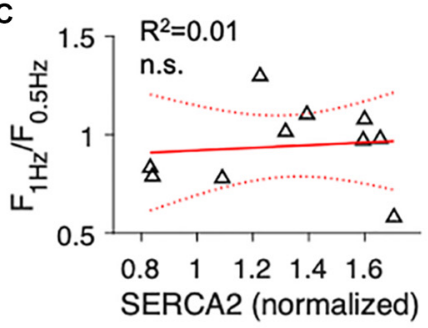

E

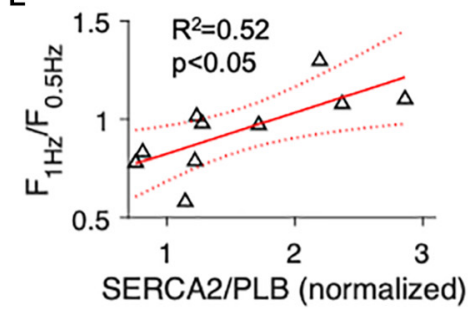

FIGURE 3 | Association of the force-frequency relationship with expression levels of proteins involved in cardiomyocyte $\mathrm{Ca}^{2+}$ cycling. (A) Example images of Western blots of cardiac sodium-calcium exchanger (NCX1), cardiac sarco/endoplasmic reticulum $\mathrm{Ca}^{2+}$-ATPase (SERCA2), total phospholamban (PLB), phospho-S16 (pS16) PLB, phospho-T17 (pT17) PLB, and glyceraldehyde 3-phosphate dehydrogenase (GAPDH). (B) Linear model showing the correlation of force at $1 \mathrm{~Hz}$ relative to force at $0.5 \mathrm{~Hz}$ pacing rate $\left(\mathrm{F}_{1 \mathrm{~Hz}} / \mathrm{F}_{0.5 \mathrm{~Hz}}\right)$ with $\mathrm{NCX} 1$ protein expression (normalized to a reference sample). $\mathrm{R}^{2}$ is the coefficient of determination, $\mathrm{P}$ indicates the probability of type I error of linear regression. Dotted lines indicate $95 \%$ confidence intervals. (C) Correlation of $\mathrm{F}_{1 \mathrm{~Hz}} / \mathrm{F}_{0.5 \mathrm{~Hz}}$ with SERCA2 protein expression. (D) Correlation of $\mathrm{F}_{1 \mathrm{~Hz}} / \mathrm{F}_{0.5 \mathrm{~Hz}}$ with total PLB protein expression. (E) Correlation of $\mathrm{F}_{1 \mathrm{~Hz}} / \mathrm{F}_{0.5 \mathrm{~Hz}}$ with the ratio of SERCA2 to total PLB.

PLBpT17/PLB ratio) predicted the FFR significantly better, but not to the same extent as $\Delta \mathrm{TT}$ (Figure 2A).

\section{T-System Loss Is Associated With Slowed Myocardial Relaxation}

We then sought to investigate parameters of contractile kinetics. Because contraction kinetics, especially relaxation time, may depend on diastolic sarcomere length (Janssen, 2010), we assessed the degree of cardiomyocyte disarray (Varnava et al., 2001) to rule out that differing fractions of non-aligned myocytes would affect the results (Supplementary Figure S4). Analyzing 2D confocal tile scans of 10 samples, we found that myocyte main axis orientation showed a standard deviation (dispersion) of $18.9 \pm 1^{\circ}$ and that $11.1 \pm 1.8 \%$ of the myocytes deviated by more than $30^{\circ}$ from the main fiber orientation. Both measures were similar in samples with high and low t-system density (Supplementary Figures S4F,G), allowing the conclusion that myocyte disarray did not bias contraction kinetics. We analyzed individual contractions recorded at $1 \mathrm{~Hz}$ stimulation frequency and determined the mean TTP, TTR, and CD90 for each sample during a $30 \mathrm{~s}$ interval. We then fitted linear models with TTP, TTR or $\mathrm{CD}_{90}$ as dependent variables and either SERCA2/PLB (Figures 4A-C) or $\triangle \mathrm{TT}$ (Figures 4D-F) as predicting parameters. TTP and TTR showed a negative trend with increasing SERCA2/PLB ratio, but statistical significance was reached for $\mathrm{CD}_{90}$ only (Figure $4 \mathrm{C}, R^{2}=0.42, p<0.05$ ). This suggests that the trends on force increase and relaxation time add up and cause a prolonged contraction duration at low SERCA activity. Similar results were obtained for the PLBpT17/PLB ratio $\left(R^{2}=0.49\right.$, data not shown). To our surprise, $\Delta \mathrm{TT}$ did not correlate with TTP (Figure 4D), but rather with TTR and CD90
( $R^{2}=0.5$ and $R^{2}=0.39$, respectively, $p<0.05$, Figures 4E,F). This prompted us to test for a relationship between NCX1 and TTR, and interactions between NCX1 and $\triangle \mathrm{TT}$, because the velocity of cytosolic $\mathrm{Ca}^{2+}$ extrusion via NCX1 may contribute to myocardial relaxation particularly in failing hearts (Hasenfuss et al., 1999). Moreover, NCX1 density has been reported to be high in t-tubules (Thomas et al., 2003). While NCX1 expression was not associated with relaxation time (Figure 4G) or $\Delta \mathrm{TT}$ $\left(R^{2}=0.1\right.$, n.s., data not shown), we found that a multivariate model including $\Delta \mathrm{TT}$ and the interaction of $\triangle \mathrm{TT}$ with NCX1 ( $\triangle$ TT.NCX) predicted TTR significantly better than NCX1 expression or $\Delta \mathrm{TT}$ alone $\left(R^{2}=0.79, p<0.01\right.$, Figure $\left.4 \mathrm{H}\right)$. The data indicate that with increasing degrees of t-system loss contraction duration is prolonged, and that the prolongation is mainly due to an increased relaxation time, which not only depends on the t-system, but also on NCX expression. This can be interpreted as an increased influence of the t-system on relaxation time when NCX expression is low.

\section{DISCUSSION}

A reduced contractile reserve is a hallmark of chronic heart failure and leads to exacerbation of symptoms especially under conditions of cardiac stress, for example during physical activity. While in healthy myocardium, the FFR contributes to adaptation of cardiac output to increased demands, the intrinsic cardiac mechanisms are blunted or even inverted in failing myocardium (Mulieri et al., 1992; Schwinger et al., 1994). Furthermore, impaired myocardial relaxation may add to reduced diastolic filling in certain types of heart failure. Although the cellular and 

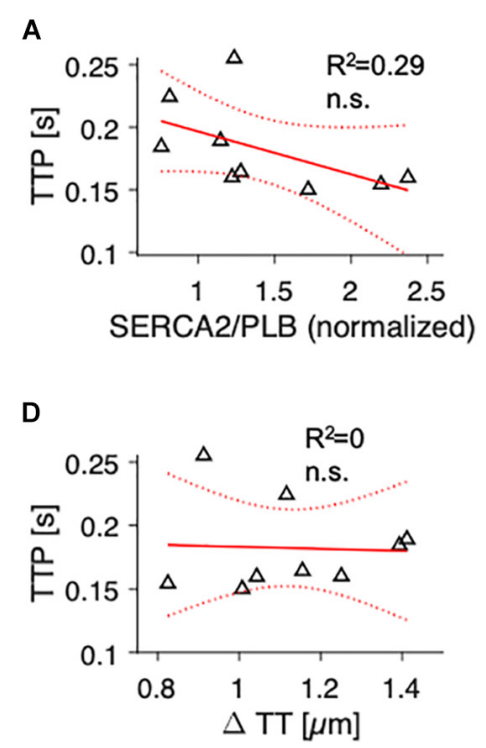
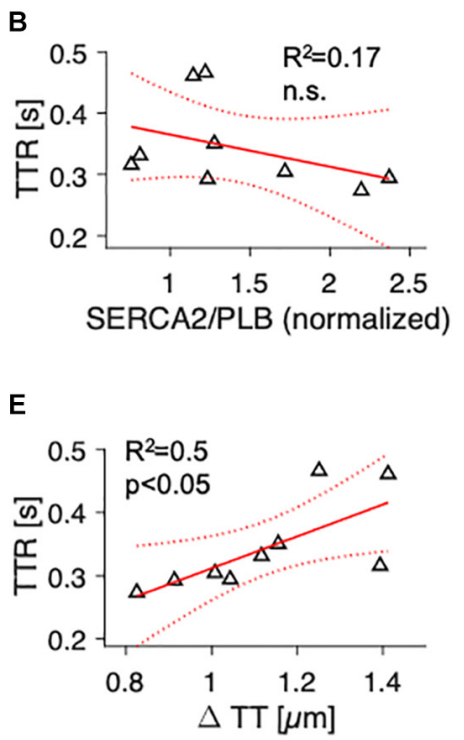

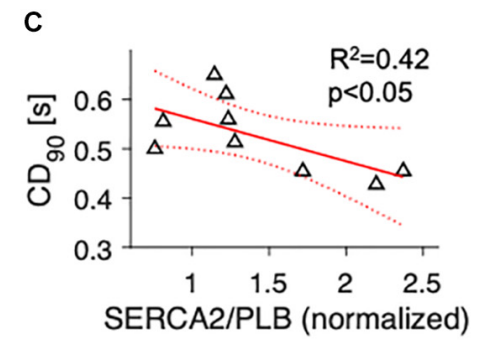

$\mathbf{F}$

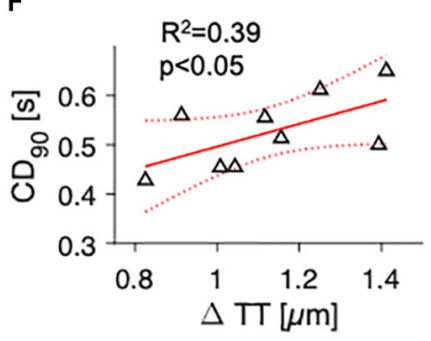

G

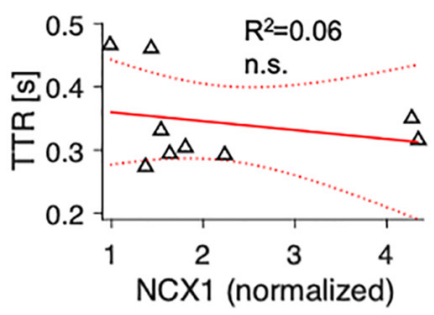

H

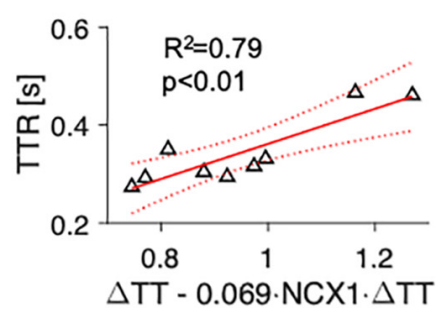

FIGURE 4 | Association of contraction parameters at $1 \mathrm{~Hz}$ pacing rate with SERCA2/PLB ratio, t-tubule distance and NCX1 expression. Linear models showing the correlation of (A) time to peak (TTP), (B) time to relaxation (TTR), and (C) $90 \%$ contraction duration (CD90) with SERCA2/PLB, and of (D) TTP, (E) TTR and (F) CD90 with t-tubule distance $(\Delta T T) . R^{2}$ is the coefficient of determination, $p$ indicates the probability of type I error of linear regression. Dotted lines indicate $95 \%$ confidence intervals. (G) Correlation of TTR with NCX1. (H) Multivariate regression model combining $\Delta T$ and the interaction of $\Delta T T$ with $N C X(\Delta T T+\Delta T$ : NCX) as predictors of TTR. The fitted model, TTR $=0.36 \cdot(\Delta T T-0.069 \cdot N C X 1 \cdot \Delta T T)$, explains the variance in TTR significantly better than simple linear models with only NCX1 $(p<0.001)$ or $\Delta T T(p=0.01$, likelihood ratio test).

molecular mechanisms leading to these functional impairments have been intensively studied, they are still not entirely clear. It is unknown if remodeling of the t-system, which commonly occurs in chronic heart failure, contributes to FFR inversion and slowing of contraction in intact myocardium from failing human hearts. Here, we describe a close correlation of t-system loss with negative FFR and altered contractile kinetics in human myocardial tissue slices, using confocal microscopy and a recently published method for biomimetic assessment and culture of human myocardium (Fischer et al., 2019). With this, we provide additional evidence that t-system remodeling is an important pathomechanism in heart failure and may predict myocardial dysfunction on a very small spatial scale.

\section{T-System and FFR}

Our results show that there was a high variability in the functional myocardial response to increasing stimulation frequency. This may be surprising, because all patients suffered from endstage heart failure with severely reduced LV ejection fraction (Supplementary Table S1), and it was shown that the FFR is blunted or reversed on the whole-heart level in heart failure patients (Hasenfuss et al., 1994a). One might therefore expect a negative FFR in all samples. However, considering the finding that myocardial contractility as well as t-system remodeling vary within failing hearts (Crossman et al., 2015), it becomes clear that samples taken randomly from the LV free wall as done in this study, show variations in structure and function. In fact, others also reported high variations in FFR amongst muscle strips obtained from failing hearts (Hasenfuss et al., 1994b). Here, we demonstrate that $\mathrm{t}$-system distance, a histological parameter measuring the degree of $\mathrm{t}$-system loss and remodeling (Seidel et al., 2017a), predicts the FFR with comparable or even higher accuracy than previously reported parameters of protein expression (Hasenfuss et al., 1994b). T-system remodeling may contribute to reduced force development at higher stimulation frequencies by causing decreased and slowed SR $\mathrm{Ca}^{2+}$ release due to impaired coupling between L-type $\mathrm{Ca}^{2+}$ channels and ryanodine receptors (Louch et al., 2004; Seidel et al., 2019). This may reduce the contractile reserve when fast $\mathrm{Ca}^{2+}$ cycling is required. 
We also found that high PLB protein expression levels were associated with a negative FFR, suggesting that SERCA inhibition hindered the force increase at high pacing rates. Consistently, a high ratio of PLBpT17 to total PLB, which may reflect SERCA activity, correlated positively with the FFR, whereas SERCA expression levels did not. This is in accordance with a study in failing and non-failing human myocardium reporting a positive correlation of the FFR with SERCA activity, but not with SERCA protein levels (Munch et al., 2000). However, it appears to be in contrast with a study demonstrating that SERCA and PLB overexpression decreased and increased the frequency response, respectively, in isolated rabbit cardiomyocytes (Meyer et al., 1999) and another study reporting that PLB knockout blunts the FFR in mouse hearts (Wu et al., 2012). A possible explanation is that in the cases of SERCA overexpression or PLB knockout $\mathrm{SR} \mathrm{Ca}^{2+}$ capacity is close to its maximum already at low pacing frequencies and therefore cannot increase at higher frequencies (Janssen and Periasamy, 2007). The degree of PLB phosphorylation at the CAMKII-specific site T17 may reflect CAMKII activity in a sample and therefore correlate positively with the FFR, as CAMKII has been implied as an important modulator of the frequency response (Zhao et al., 2004; Wu et al., 2012). However, considering that CAMKII is activated by cytosolic $\mathrm{Ca}^{2+}$, it seems as well possible that increased fractions of PLBpT17 indicate higher intracellular $\mathrm{Ca}^{2+}$ levels, which in turn could result from higher t-system density. This becomes clear from the large contribution of the $\mathrm{t}$-system to membrane surface area (Despa et al., 2003; Pasek et al., 2008) and a particularly high density of L-type $\mathrm{Ca}^{2+}$ channels in t-tubules (Brette et al., 2006; Seidel et al., 2019). Thus, assuming that L-type $\mathrm{Ca}^{2+}$ current density is unaltered or even decreased in samples with low t-system density, a reduction in membrane surface would result in diminished $\mathrm{Ca}^{2+}$ influx and might limit the accumulation of intracellular $\mathrm{Ca}^{2+}$ required for a positive FFR, especially when the contribution from SR $\mathrm{Ca}^{2+}$ is low (Stemmer and Akera, 1986; Endoh, 2004). This mechanism could link t-system density to CAMKII activity, but its investigation would require freezing of the tissue slices immediately after pacing with different frequencies. Here, we quantified PLBpT17 levels at rest only. PLB and the PLBp17/PLB ratio correlated moderately with $\Delta \mathrm{TT}\left(R^{2}=0.48\right.$ and 0.37 , respectively). However, dividing the cohort into samples with low and high PLB levels, using the corresponding median as threshold, still yielded high correlations of $\Delta$ TT with the FFR $\left(R^{2}>0.7, p<0.05\right)$, suggesting that the t-system and resting PLBpT17/PLB levels are independently related to the FFR.

The finding that $\mathrm{t}$-system loss predicts negative FFR, which translates into reduced myocardial wall tension at high pacing rates (Figure 1E), fits well to results from other studies. Acutely detubulated rat myocardium showed a blunted FFR (Ferrantini et al., 2014). Assuming that SERCA and PLB are not affected by detubulation, this may additionally indicate that the dependence of a positive FFR on high t-system density is not confounded by altered SERCA activity in samples with $\mathrm{t}$-system loss. Another study showed that the FFR is flat in human newborns and becomes positive in infants. At the same time, $\mathrm{t}$-tubules develop in ventricular cardiomyocytes
(Wiegerinck et al., 2009). Collectively, these findings support the hypothesis that the t-system is required for a positive FFR in human ventricular cardiomyocytes.

\section{T-System and Contractile Kinetics}

In addition to the relationship between the t-system and FFR, we found that $\mathrm{t}$-system loss was associated with slowed contractile kinetics. TTP, however, did not correlate with $\Delta \mathrm{TT}$, although acute detubulation was reported to prolong the twitch peak time in rat ventricular trabeculae (Ferrantini et al., 2014). It is possible that due to slower contraction velocity in human than rat hearts (Milani-Nejad and Janssen, 2014), contraction velocity in the rat depends more steeply on the t-system. Moreover, t-system density in rat myocytes is markedly higher than in normal human myocytes (Jayasinghe et al., 2012). T-system loss in rat cardiomyocytes may thus lead to a more pronounced decrease in contraction velocity. Here, a relationship between $\Delta \mathrm{TT}$ and TTP was not detectable, possibly because of varying age, underlying disease or drug therapy in the samples. Another factor that could affect contractile kinetics is resting sarcomere length (Janssen, 2010). Although we applied a defined diastolic preload to all samples, and found that the degree of myocyte disarray was similar in samples with low and high $\mathrm{t}$-system density (Supplementary Figures S2, S4), we cannot fully exclude that variability stemming from tissue preparation may have masked a possible correlation between $\Delta \mathrm{TT}$ and TTP. Nevertheless, we did find that $\Delta \mathrm{TT}$ predicted relaxation time and overall contraction duration. This is consistent with slowed contraction as well as slowed relaxation time observed in acutely detubulated rat myocardium (Ferrantini et al., 2014). As an underlying mechanism we suggest a slowed decay of the intracellular $\mathrm{Ca}^{2+}$ transient, because $\mathrm{Ca}^{2+}$ removal via NCX is hindered when t-system density is low (Thomas et al., 2003; Ferrantini et al., 2014). This effect may be especially pronounced in human failing myocardium, where the relative contribution of NCX to $\mathrm{Ca}^{2+}$ removal seems to be increased because of SERCA downregulation (Hasenfuss et al., 1999). It is well possible that an intact t-system is required for efficient NCX function because the diffusion of intracellular $\mathrm{Ca}^{2+}$ ions to the surface sarcolemma may take much longer than to intracellularly located $\mathrm{t}$-tubules. If these are lost, $\mathrm{Ca}^{2+}$ extrusion will consequently be slowed, leading to prolonged relaxation time. We provide some evidence for this idea by showing that a linear model taking into account the interaction of $\triangle \mathrm{TT}$ with $\mathrm{NCX}$ predicted the relaxation time significantly better than $\triangle \mathrm{TT}$ or NCX alone. The negative interaction term in the resulting model points to an increased influence of $\triangle \mathrm{TT}$ on relaxation time when NCX expression is low. Thus, increased expression levels of NCX found in failing myocardium (Hasenfuss et al., 1996, 1999) might compensate not only for reduced SERCA activity, but also for t-system loss. Based on these exploratory findings we propose a role of the $t$-system in conjunction with NCX for myocardial relaxation. However, this hypothesis will have to be tested in future studies.

\section{Limitations}

The heart failure patients from whom samples were obtained in this study were heterogeneous regarding etiology, 
pharmacological treatments and concomitant diseases. Thus, although, the sample size used here $(n=13)$ was comparable to that of other studies (Mulieri et al., 1992; Hasenfuss et al., 1994b; Munch et al., 2000), a larger number of samples would be required to increase statistical power for subgroup analyses, to investigate the influence of clinical parameters on the functional and histological myocardial parameters, and to increase robustness against statistical outliers. However, despite the heterogeneity of the cohort, the main results, that is, correlation of $\mathrm{t}$-system loss with negative FFR and, in conjunction with NCX1 expression, the prediction of slowed myocardial relaxation by t-system loss, showed high statistical significance. This was possibly reached because the structure-function relationships were assessed on a millimeter scale. It remains to be determined if $\mathrm{t}$-system remodeling may predict cardiac function also on larger scales and how t-system remodeling varies across failing hearts.

\section{DATA AVAILABILITY STATEMENT}

The datasets generated for this study are available on request to the corresponding author.

\section{ETHICS STATEMENT}

The studies involving human participants were reviewed and approved by the Institutional Review Boards of the University of Erlangen-Nuremberg, the Ruhr-University Bochum and the Ludwig Maximilian University of Munich. The patients/participants provided their written informed consent to participate in this study.

\section{REFERENCES}

Brette, F., Salle, L., and Orchard, C. H. (2006). Quantification of calcium entry at the T-tubules and surface membrane in rat ventricular myocytes. Biophys. J. 90, 381-389. doi: 10.1529/biophysj.105.069013

Brixius, K., Wollmer, A., Bolck, B., Mehlhorn, U., and Schwinger, R. H. (2003). Ser16-, but not Thr17-phosphorylation of phospholamban influences frequency-dependent force generation in human myocardium. Pflugers Arch. 447, 150-157. doi: 10.1007/s00424-003-1163-3

Crossman, D. J., Young, A. A., Ruygrok, P. N., Nason, G. P., Baddelely, D., Soeller, C., et al. (2015). T-tubule disease: relationship between t-tubule organization and regional contractile performance in human dilated cardiomyopathy. J. Mol. Cell Cardiol. 84, 170-178. doi: 10.1016/j.yjmcc.2015.04.022

Despa, S., Brette, F., Orchard, C. H., and Bers, D. M. (2003). Na/Ca exchange and $\mathrm{Na} / \mathrm{K}$-ATPase function are equally concentrated in transverse tubules of rat ventricular myocytes. Biophys. J. 85, 3388-3396. doi: 10.1016/S0006-3495(03) $74758-74754$

Endoh, M. (2004). Force-frequency relationship in intact mammalian ventricular myocardium: physiological and pathophysiological relevance. Eur. J. Harmacol. 500, 73-86. doi: 10.1016/j.ejphar.2004.07.013

Ferrantini, C., Coppini, R., Sacconi, L., Tosi, B., Zhang, M. L., Wang, G. L., et al. (2014). Impact of detubulation on force and kinetics of cardiac muscle contraction. J. Gen. Physiol. 143, 783-797. doi: 10.1085/jgp.2013 11125

\section{AUTHOR CONTRIBUTIONS}

TS, TV, AD, CH, MW, and HM contributed to the conception and design. MA-K, DF, SS, GM, RT, and TS contributed to the data acquisition and experiments. TS, MA-K, DF, and RT contributed to the data analysis. TS, TV, and MA-K contributed to the interpretation of the data. MA-K and TS contributed to the drafting of the manuscript. All authors revision and approval of the manuscript.

\section{FUNDING}

This work was supported by the DZHK (German Centre for Cardiovascular Research) and by the Interdisciplinary Centre for Clinical Research (IZKF) at the University Hospital of the University of Erlangen-Nuremberg.

\section{ACKNOWLEDGMENTS}

For excellent technical support we would like to thank Celine Grüninger and Lorenz McCargo from the Institute of Cellular and Molecular Physiology Erlangen as well as Caroline Stanasiuk and Desiree Gerdes from the Heart and Diabetes Center NRW. We also thank the Universitätsbund Erlangen-Nürnberg for financially supporting the vibratome.

\section{SUPPLEMENTARY MATERIAL}

The Supplementary Material for this article can be found online at: https://www.frontiersin.org/articles/10.3389/fphys. 2020.00182/full\#supplementary-material

Fischer, C., Milting, H., Fein, E., Reiser, E., Lu, K., Seidel, T., et al. (2019). Long-term functional and structural preservation of precision-cut human myocardium under continuous electromechanical stimulation in vitro. Nat. Commun. 10:117. doi: 10.1038/s41467-018-08003-1

Hasenfuss, G., Holubarsch, C., Hermann, H. P., Astheimer, K., Pieske, B., and Just, H. (1994a). Influence of the force-frequency relationship on haemodynamics and left ventricular function in patients with non-failing hearts and in patients with dilated cardiomyopathy. Eur. Heart. J. 15, 164-170. doi: 10.1093/ oxfordjournals.eurheartj.a060471

Hasenfuss, G., Reinecke, H., Studer, R., Meyer, M., Pieske, B., Holtz, J., et al. (1994b). Relation between myocardial function and expression of sarcoplasmic reticulum $\mathrm{Ca}(2+)$-ATPase in failing and nonfailing human myocardium. Circ. Res. 75, 434-442. doi: 10.1161/01.res.75.3.434

Hasenfuss, G., Reinecke, H., Studer, R., Pieske, B., Meyer, M., Drexler, H., et al. (1996). Calcium cycling proteins and force-frequency relationship in heart failure. Basic Res. Cardiol. 91(Suppl. 2), 17-22. doi: 10.1007/bf00795357

Hasenfuss, G., Schillinger, W., Lehnart, S. E., Preuss, M., Pieske, B., Maier, L. S., et al. (1999). Relationship between $\mathrm{Na}+-\mathrm{Ca} 2+$-exchanger protein levels and diastolic function of failing human myocardium. Circulation 99, 641-648. doi: 10.1161/01.cir.99.5.641

Higginbotham, M. B., Morris, K. G., Williams, R. S., McHale, P. A., Coleman, R. E., and Cobb, F. R. (1986). Regulation of stroke volume during submaximal and maximal upright exercise in normal man. Circ. Res. 58, 281-291. doi: 10.1161/01.res.58.2.281 
Janssen, P. M. (2010). Myocardial contraction-relaxation coupling. Am. J. Physiol. Heart Circ. Physiol. 299, H1741-H1749. doi: 10.1152/ajpheart.00759.2010

Janssen, P. M., and Periasamy, M. (2007). Determinants of frequency-dependent contraction and relaxation of mammalian myocardium. J. Mol. Cell. Cardiol. 43, 523-531. doi: 10.1016/j.yjmcc.2007.08.012

Jayasinghe, I., Crossman, D., Soeller, C., and Cannell, M. (2012). Comparison of the organization of T-tubules, sarcoplasmic reticulum and ryanodine receptors in rat and human ventricular myocardium. Clin. Exp. Pharmacol. Physiol. 39, 469-476. doi: 10.1111/j.1440-1681.2011.05578.x

Kostin, S., Scholz, D., Shimada, T., Maeno, Y., Mollnau, H., Hein, S., et al. (1998). The internal and external protein scaffold of the T-tubular system in cardiomyocytes. Cell Tissue Res. 294, 449-460. doi: 10.1007/s004410051196

Lackey, D. P., Carruth, E. D., Lasher, R. A., Boenisch, J., Sachse, F. B., and Hitchcock, R. W. (2011). Three-dimensional modeling and quantitative analysis of gap junction distributions in cardiac tissue. Ann. Biomed. Eng. 39, 2683-2694. doi: 10.1007/s10439-011-0369-3

Louch, W. E., Bito, V., Heinzel, F. R., Macianskiene, R., Vanhaecke, J., Flameng, W., et al. (2004). Reduced synchrony of Ca2+ release with loss of T-tubules-a comparison to $\mathrm{Ca} 2+$ release in human failing cardiomyocytes. Cardiovasc. Res. 62, 63-73. doi: 10.1016/j.cardiores.2003.12.031

McDonald, R. L., Colyer, J., and Harrison, S. M. (2000). Quantitative analysis of $\mathrm{Na}+\mathrm{Ca} 2+$ exchanger expression in guinea-pig heart. Eur. J. Biochem. 267, 5142-5148. doi: 10.1046/j.1432-1327.2000.01579.x

Meyer, M., Bluhm, W. F., He, H., Post, S. R., Giordano, F. J., Lew, W. Y., et al. (1999). Phospholamban-to-SERCA2 ratio controls the force-frequency relationship. Am. J. Physiol. 276, H779-H785. doi: 10.1152/ajpheart.1999.276. 3.H779

Milani-Nejad, N., and Janssen, P. M. (2014). Small and large animal models in cardiac contraction research: advantages and disadvantages. Pharmacol. Ther. 141, 235-249. doi: 10.1016/j.pharmthera.2013.10.007

Mulieri, L. A., Hasenfuss, G., Leavitt, B., Allen, P. D., and Alpert, N. R. (1992). Altered myocardial force-frequency relation in human heart failure. Circulation 85, 1743-1750. doi: 10.1161/01.cir.85.5.1743

Munch, G., Bolck, B., Brixius, K., Reuter, H., Mehlhorn, U., Bloch, W., et al. (2000). SERCA2a activity correlates with the force-frequency relationship in human myocardium. Am. J. Physiol. Heart Circ. Physiol. 278, H1924-H1932. doi: 10.1152/ajpheart.2000.278.6.H1924

Pasek, M., Brette, F., Nelson, A., Pearce, C., Qaiser, A., Christe, G., et al. (2008). Quantification of t-tubule area and protein distribution in rat cardiac ventricular myocytes. Prog. Biophys. Mol. Biol. 96, 244-257. doi: 10.1016/j. pbiomolbio.2007.07.016

Rossman, E. I., Petre, R. E., Chaudhary, K. W., Piacentino, V. III, Janssen, P. M., Gaughan, J. P., et al. (2004). Abnormal frequency-dependent responses represent the pathophysiologic signature of contractile failure in human myocardium. J. Mol. Cell Cardiol. 36, 33-42. doi: 10.1016/j.yjmcc.2003. 09.001

Schwinger, R. H., Bohm, M., Koch, A., Schmidt, U., Morano, I., Eissner, H. J., et al. (1994). The failing human heart is unable to use the Frank-Starling mechanism. Circ. Res. 74, 959-969. doi: 10.1161/01.res.74.5.959

Seidel, T., Dräbing, T., Seemann, G., and Sachse, F. B. (2013). A semi-automatic approach for segmentation of three-dimensional microscopic image stacks of cardiac tissue. Lect. Notes Comput. Sci. 7945, 300-307. doi: 10.1007/978-3-64238899-6_36

Seidel, T., Edelmann, J. C., and Sachse, F. B. (2016). Analyzing remodeling of cardiac tissue: a comprehensive approach based on confocal microscopy and 3D reconstructions. Ann. Biomed. Eng. 44, 1436-1448. doi: 10.1007/s10439-0151465-6

Seidel, T., Fiegle, D. J., Baur, T. J., Ritzer, A., Nay, S., Heim, C., et al. (2019). Glucocorticoids preserve the t-tubular system in ventricular cardiomyocytes by upregulation of autophagic flux. Basic Res. Cardiol. 114:47. doi: 10.1007/s00395019-0758-756

Seidel, T., Navankasattusas, S., Ahmad, A., Diakos, N. A., Xu, W. D., TristaniFirouzi, M., et al. (2017a). Sheet-like remodeling of the transverse tubular system in human heart failure impairs excitation-contraction coupling and functional recovery by mechanical unloading. Circulation 135, 1632-1645. doi: 10.1161/CIRCULATIONAHA.116.024470

Seidel, T., Sankarankutty, A. C., and Sachse, F. B. (2017b). Remodeling of the transverse tubular system after myocardial infarction in rabbit correlates with local fibrosis: a potential role of biomechanics. Prog. Biophys. Mol. Biol. 130(Pt B), 302-314. doi: 10.1016/j.pbiomolbio.2017.07.006

Stemmer, P., and Akera, T. (1986). Concealed positive force-frequency relationships in rat and mouse cardiac muscle revealed by ryanodine. Am. J. Physiol. 251(6 Pt 2), H1106-H1110. doi: 10.1152/ajpheart.1986.251.6. H1106

Thomas, M. J., Sjaastad, I., Andersen, K., Helm, P. J., Wasserstrom, J. A., Sejersted, O. M., et al. (2003). Localization and function of the $\mathrm{Na}+/ \mathrm{Ca} 2+-$ exchanger in normal and detubulated rat cardiomyocytes. J. Mol. Cell Cardiol. 35, 1325-1337. doi: 10.1016/j.yjmcc.2003.08.005

Varnava, A. M., Elliott, P. M., Mahon, N., Davies, M. J., and McKenna, W. J. (2001). Relation between myocyte disarray and outcome in hypertrophic cardiomyopathy. Am. J. Cardiol. 88, 275-279. doi: 10.1016/s0002-9149(01) 01640-x

Watson, S. A., Duff, J., Bardi, I., Zabielska, M., Atanur, S. S., Jabbour, R. J., et al. (2019). Biomimetic electromechanical stimulation to maintain adult myocardial slices in vitro. Nat. Commun. 10:2168. doi: 10.1038/s41467-01910175-3

Wiegerinck, R. F., Cojoc, A., Zeidenweber, C. M., Ding, G., Shen, M., Joyner, R. W., et al. (2009). Force frequency relationship of the human ventricle increases during early postnatal development. Pediatr. Res. 65, 414-419. doi: 10.1203/ PDR.0b013e318199093c

Wu, Y., Luczak, E. D., Lee, E. J., Hidalgo, C., Yang, J., Gao, Z., et al. (2012). CaMKII effects on inotropic but not lusitropic force frequency responses require phospholamban. J. Mol. Cell Cardiol. 53, 429-436. doi: 10.1016/j.yjmcc. 2012.06.019

Zhao, W., Uehara, Y., Chu, G., Song, Q., Qian, J., Young, K., et al. (2004). Threonine-17 phosphorylation of phospholamban: a key determinant of frequency-dependent increase of cardiac contractility. J. Mol. Cell Cardiol. 37, 607-612. doi: 10.1016/j.yjmcc.2004.05.013

Conflict of Interest: The authors declare that the research was conducted in the absence of any commercial or financial relationships that could be construed as a potential conflict of interest.

Copyright (๑) 2020 Abu-Khousa, Fiegle, Sommer, Minabari, Milting, Heim, Weyand, Tomasi, Dendorfer, Volk and Seidel. This is an open-access article distributed under the terms of the Creative Commons Attribution License (CC BY). The use, distribution or reproduction in other forums is permitted, provided the original author(s) and the copyright owner(s) are credited and that the original publication in this journal is cited, in accordance with accepted academic practice. No use, distribution or reproduction is permitted which does not comply with these terms. 